DESIGN AND DEVELOPMENT OF A MULTILAYERED SECURITY SYSTEM

\title{
NEERAJ ADURI
}

Sreenidhi Institute of Science and Technology- Hyderabad, Telangana, India

ABSTRACT
The aim of this design is to provide a multi-level security system to prevent entry of individuals in restricted
areas. In this paper, we deal with a simple two-layer design, which is implemented using a Keypad code (level 1) and a
One Time Password (OTP) that will be shared to the registered mobile number which has to be entered (level 2) in order
to unlock a safe. In this particular design, the implementation is carried out by using a Micro controller. We are going
for a multilayer security system to enhance the security, and this can be extended further by adding another layer or
even more to make it impeccable. We used a $4 * 4$ keypad as a medium to enter our keypad code and encrypted password,
and we used Atmegal6 as the micro controller for programming the code.
KEYWORDS: Micro Controller (Atmega 16), Encryption \& One Time Password

Received: Apr 24, 2017; Accepted: May 20, 2017; Published: Jun 03, 2017; Paper Id.: IJECIERDJUN20171

INTRODUCTION

In the first stage of design, just like any other card PIN, each user is given a unique four-digit number. The user has to enter the code correctly via a keypad, and failing to do so, will result in throwing an error message on the $16 * 2$ LCD display screen that we are using. Then, the user has to try and enter the password correctly, once again. If the user has entered the password correctly, then a success message would be displayed on the screen and a new message will be displayed on the screen, which will asks the user to click on a particular button that generates the OTP, which is to be shared to the user's mobile using a GSM module. This is a 5-digit number and the user has to use the same keypad to enter the OTP that he had received. Here, we use the concept of timers to generate that OTP.

Here, if we look into the process, it is palpable that the implementation of this design poses many challenges. First of all, we need to interface the GSM Module with the micro controller. USART communication is used to interface the micro controller with the GSM module. RS-232 cable is used to establish this connection between the two modules. Also, we need to use a transformer circuit to pull down the Voltage ranges from regular $240 \mathrm{~V}$ to $5 \mathrm{~V}$, as the Micro-controller being used cannot withstand such high voltages. In addition, we need to code the Micro controller timers by triggering the Timer/Counter Control Register (TCCR) of the Atmega16. Atmega16 gives us a comfort in this regard; as it has inbuilt Timer, Counter and USART functionalities and these can be activated by programming those particular registers.

\section{DESIGN FLOW}

The following block diagram gives us an understanding of the flow of the process and the series of events that are going to take place. 


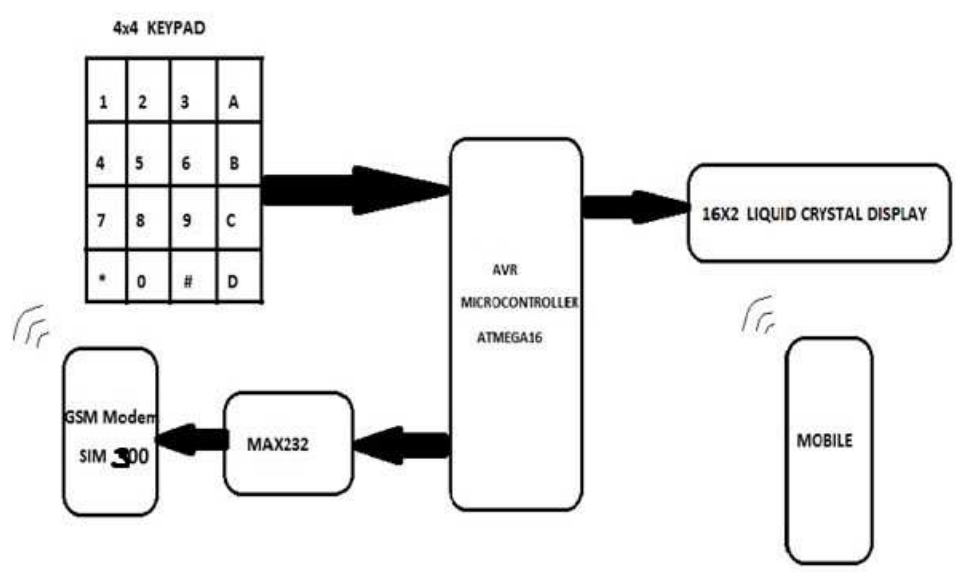

Figure 1

Here, the GSM Module must have a SIM card and just like any other message that we sent, we can trigger a message (OTP) from this module and that can be sent to the registered mobile number.

The microcontroller can communicate with the serial devices using its single Serial Port. The logic levels at which this serial port operates is TTL logics. But, some of the serial devices operate at RS 232 Logic levels. So, in order to communicate the Microcontroller with either GSM modem or PC, a mismatch between the Logic levels occurs. In order to avoid this mismatch, a Serial driver is used. And MAX 232 is a Serial Line Driver used to establish communication between microcontroller and PC (or GSM).

\section{CIRCUIT DESIGN}

Micro-controller that we are using has 4 ports viz., PORTA, PORTB, PORTC, PORTD and each PORT has little additional functionality apart from acting as a basic input and output PORT. PORTD has the TX and Rx (Transmitter and Receiver) pins that facilitate the USART communication and we are using PORTB and PORTA for interfacing the keypad and LCD display, respectively. Also, as we need a button to generate the OTP, we are going to use one PIN of PORTC (C7). An important thing here is we need to ground the A Vcc and Aref pins of Atmega16 so that the ADC functionality that PORTA has is not activated.

The following shows us a schematic diagram of the implementation and apart from GSM Module that has to be connected to the RS232 every other detail has been clearly given. 


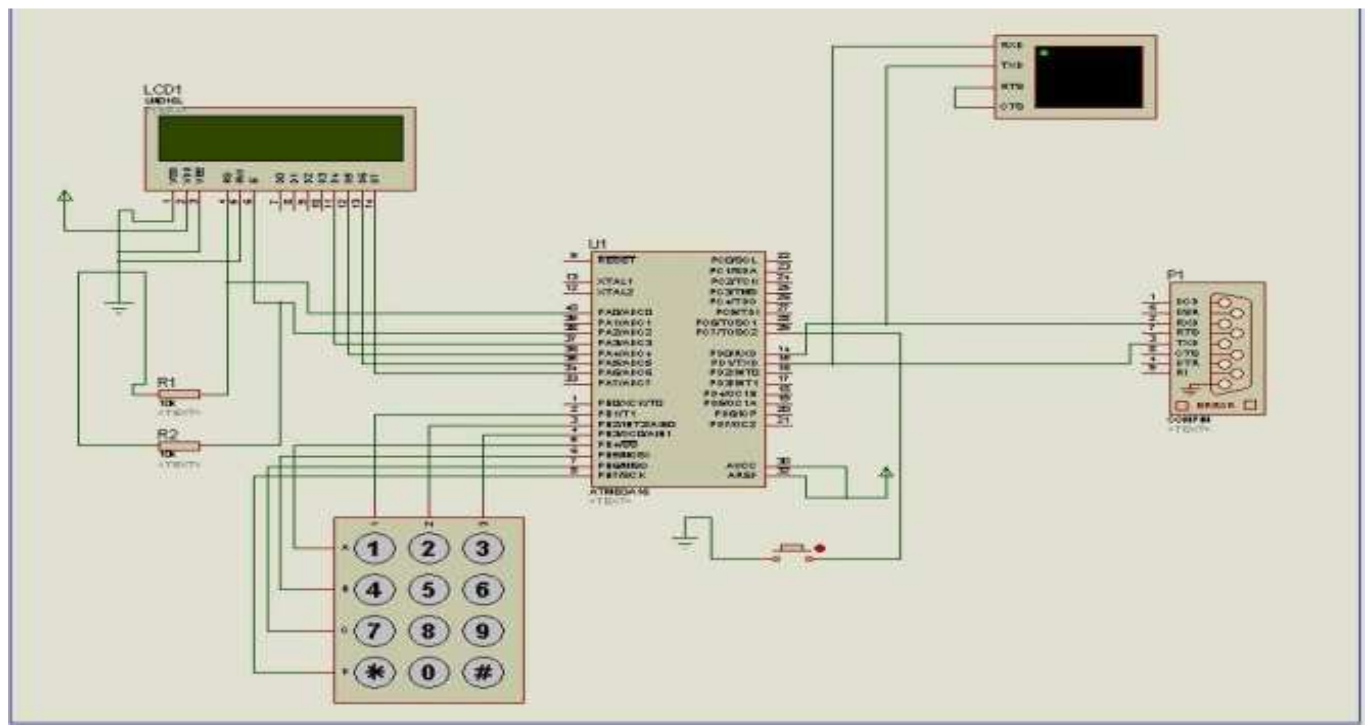

Figure 2

This is a schematic design of the circuit and this had been generated in a simulation software tool, called Proteus.

It is very important that we simulate the design and verify, if everything is working fine. Only then we proceed to burn the code in the ROM of Atmega16. Simulation tools like these help a lot in verifying the code as we can identify if there is an error in the code by seeing the simulation results.

\section{Note}

- In the above design, we have connected a $3 * 4$ Keypad but the real design has been implemented by using a $4 * 4$ keypad. This does not make any difference as the $4^{\text {th }}$ column of the keypad will have alphabets $(A, B, C, D)$ which we are not going to use.

- While interfacing the GSM module with the RS232 we need to keep in mind of the fact that tax pin of GSM module has to be connected to Rx pin of RS232 and Rx pin of the GSM module has two connected to TX pin of RS232.

\section{PROGRAMMING THE MICRO CONTROLLER}

This is the Herculean task of the project. We can download the datasheet of the Micro-controller and we have to study it, in order to know the functionalities of each of the registers. We can use the already existing header files such as LCD. h, LCD. c, gsm. c, utilsdelay. h, avr /io.h. However, we need to complete code to implement the working of the keypad. Also, we need to code to generate the OTP which has a key role in providing the security. As discussed earlier whenever the PIN C7 is triggered, an input pulse is sent to the Micro controllers processing unit. We have used the TCCR0 register and we will have already generated a sawtooth waveform. The height of this waveform $\mathrm{r}$ ranges from $0-32767$. We can decide on the type of waveform, and also the clock cycle by programming the appropriate bits of the TCCR0 register. Whenever the trigger is received by the processing unit, it cuts the saw tooth signal and note downs the value of the magnitude of the so generated waveform. This becomes the OTP. As the Micro-controller will be operating with $1 \mathrm{Mz}$ frequency, it becomes impossible to crack the code by simple means. This generated code is sent to the GSM module through USART and that will be sent as a text message to the user. 
This code that has been written is tested in a simulation tool, and only then it is moved to the hardware design. If the code is all good at the simulation, we need to burn the code into the micro controller and for this; we have needed a software tool called Sinaprog. Sinaprog is open software, and this can be used to flash the code into the ROM of all the AVR Microcontrollers.

\section{RESULTS}

The design is planned and implemented by assembling it on the bare vector board. After assembling the circuit, check the connections before switching ON the power supply.

When the power is switched on, the LCD displays the following data.

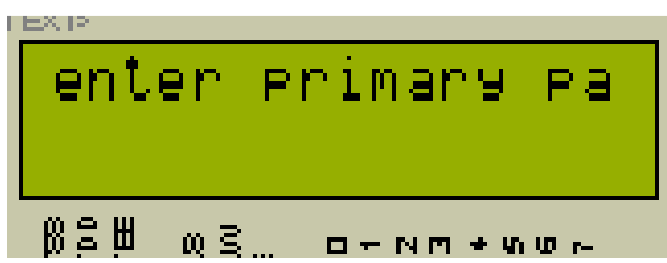

Figure 3

After the entry of the primary password, the LCD displays the following data.

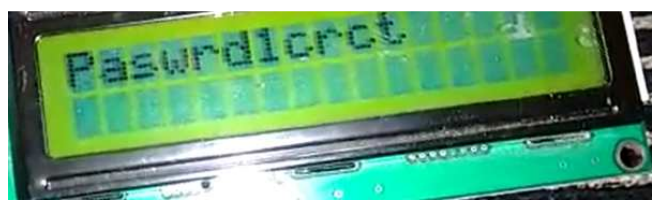

Figure 4

After primary password verification, the following is displayed on LCD screen
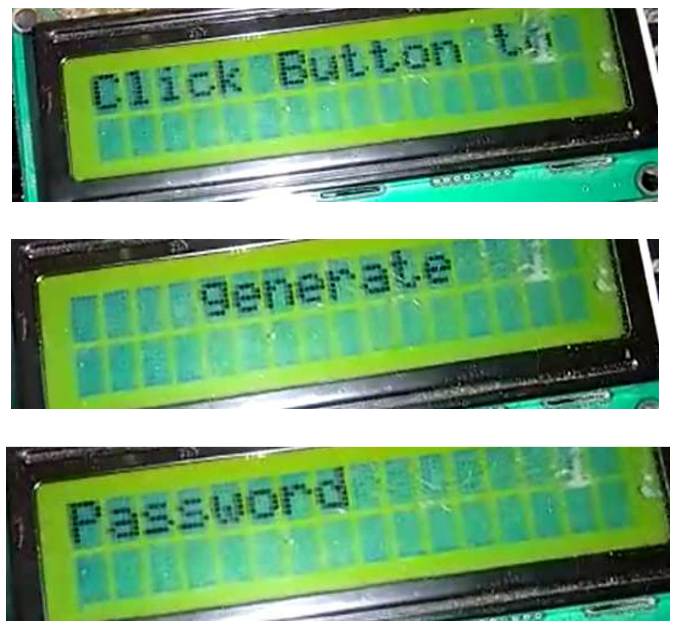

Figure 5

When the push button is pressed, the following is displayed. 


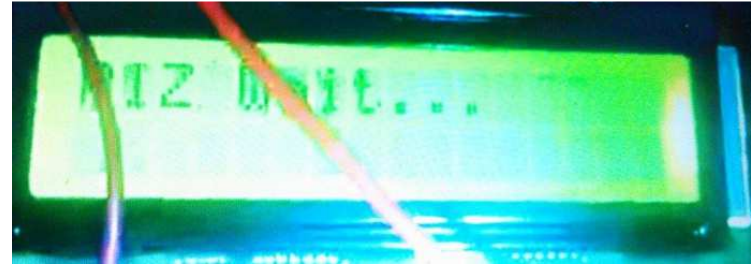

Figure 6

If the GSM fails to send the message, the following data is displayed

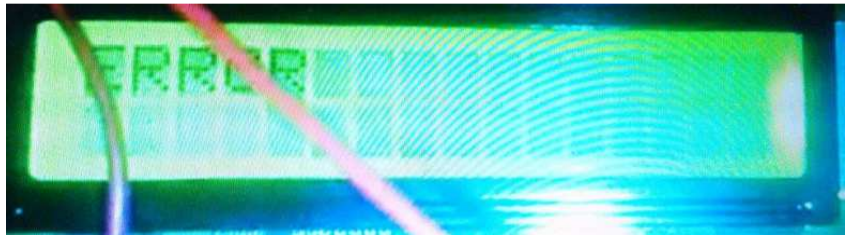

Figure 7

If the message is sent successfully, the following is displayed

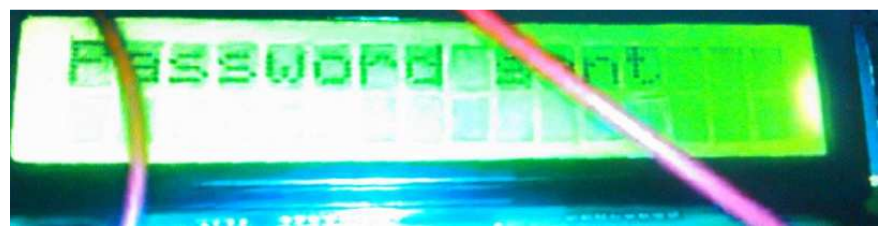

Figure 8

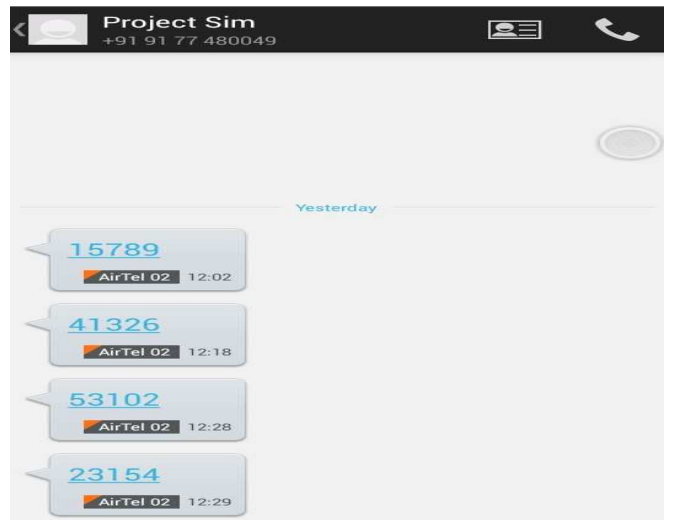

Figure 9

Then, the password sent to the phone has to be entered, If the password entered is correct, the following message is displayed.

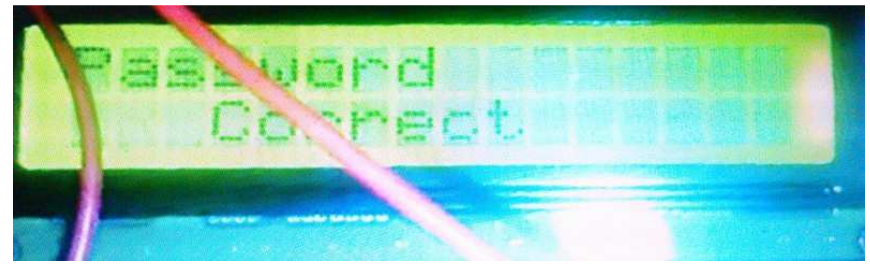

Figure 10 


\section{LIMITATIONS}

The only limitation of this design is the use of mobile communication. In this era, where mobile has dominated the likes of laptops, this design seems to be the perfect fit; however, for this design to work, the user and the GSM module we are using has to be in the area, where, the signal strength for cellular communication is strong.

\section{CONCLUSIONS}

Security provision is the main concern in this project. OTP provides this security system at low-cost. As the project is associated with a GSM modem, the SMS alert mechanism makes it very much secure, so as to act instantaneously. This is a fundamental design and this has been carried out in 2 layers. This can be further extended by adding finger print detection, by using RFID tags, etc. In today's world, where automation has become the new trend, designs like these would help in achieving this goal.

\section{REFERENCES}

1. Atmega16 data sheet given by Atmel Corporation.

2. 'AVR Microcontroller and Embedded Systems: Using Assembly and C' by Muhammad Ali Mazidi, Sarmad Naimi and Sepehr Naimi.

3. Extreme electronics.co from where I was able to get all the header files that I have used.

4. Wireless Networking Absolute Beginner's Guide by Michael Miller (2013).

5. Wireless Networks by Clint Smith and Daniel Collins (2014). 